\title{
Upregulation of LINC00659 expression predicts a poor prognosis and promotes migration and invasion of gastric cancer cells
}

\author{
PIHAI GONG ${ }^{1,2}$, YING XU ${ }^{2}$, MIN LIU $^{2}$, XIAOHUI SHEN $^{1}$, YUHANG MAO ${ }^{2}$, \\ YIPING $\mathrm{LI}^{3}$, KUN ZHANG ${ }^{4}$, SHENLING YU $^{2}$ and HONG FAN ${ }^{1}$ \\ ${ }^{1}$ Department of Medical Genetics and Developmental Biology, Medical School of Southeast University, \\ Key Laboratory of Developmental Genes and Human Diseases, Ministry of Education, Southeast University, \\ Nanjing, Jiangsu 210009; ${ }^{2}$ School of Life Science and Technology, Southeast University, Nanjing, Jiangsu 210018; \\ ${ }^{3}$ Department of Pathology, Medical School of Southeast University, Nanjing, Jiangsu 210009; ${ }^{4}$ Department of Medicine, \\ The Third Affiliated Hospital of Harbin Medical University, Harbin, Heilongjiang 154000, P.R. China
}

Received August 25, 2020; Accepted February 25, 2021

DOI: $10.3892 / \mathrm{ol} .2021 .12818$

\begin{abstract}
Long non-coding RNAs (lncRNAs) serve an important role in the progression of cancer. LINC00659 was recently identified as a novel oncogenic lncRNA involved in colon cancer cell proliferation via modulating the cell cycle. However, the function of LINC00659 in other types of cancer, especially in gastric cancer (GC), remains unknown. In the present study, bioinformatics analysis combined with cell experiments were performed to explore the function of LINC00659 in GC. It was revealed that LINC00659 expression was significantly upregulated in GC tissues and cell lines. Increased levels of LINC00659 were associated with advanced tumor stage and unfavorable prognosis of patients with GC. Additionally, upregulated LINC00659 expression promoted the migration and invasion of GC cells. Further analysis using a bioinformatics method revealed that matrix metalloproteinase 15 and IQ motif-containing GTPase activating protein 3 were potential downstream targets of LINC00659 involved in tumor metastasis, although the precise underlying mechanism requires further exploration.
\end{abstract}

\section{Introduction}

Gastric cancer (GC) is one of the most commonly occurring malignancies worldwide, and its incidence and mortality rates rank fifth and second, respectively, among all types of cancer

Correspondence to: Professor Hong Fan, Department of Medical Genetics and Developmental Biology, Medical School of Southeast University, Key Laboratory of Developmental Genes and Human Diseases, Ministry of Education, Southeast University, 87 Dingjiaqiao Road, Nanjing, Jiangsu 210009, P.R. China

E-mail: fanh@seu.edu.cn

Key words: long non-coding RNA, cell migration, cell invasion, gastric cancer, prognosis according to the Global Cancer Statistics 2018 (1). The 5-year overall survival (OS) rate of patients with $\mathrm{GC}$ is $<20 \%$, and this low survival rate is partly due to a lack of effective early diagnostic methods and prognostic indicators (2). In different types of cancer, genomic instability is associated with chromosomal aberrations, which affect numerous genes, further promoting tumor progression (3). Comparative genomic hybridization $(\mathrm{CGH})$ data have shown that gains of DNA copy number are observed frequently at chromosomes 1q, 3q, 5p, $7 \mathrm{p}, 7 \mathrm{q}, 8 \mathrm{q}, 11 \mathrm{q}, 17 \mathrm{q}, 20 \mathrm{p}$ and $20 \mathrm{q}$, and losses of one copy are frequently detected at chromosomes $3 \mathrm{p}, 4 \mathrm{p}, 4 \mathrm{q}, 8 \mathrm{p}, 9 \mathrm{p}, 18 \mathrm{p}$ and $18 q$ in GC (4-9). Several chromosomal aberrations, particularly amplification of 20q, have been observed in various types of cancer, including pancreatic $(10)$, breast $(11,12)$, colon $(13,14)$ and stomach cancer (15), implying that gain of $20 \mathrm{q}$ may have a vital role in tumorigenesis.

Amplified genes on chromosome $20 \mathrm{q}$ contain various functionally important genes involved in cell cycle regulation (E2F1, TPX2, KIF3B, PIGT and B4GALT5), nuclear function (CSEL1), viral replication (PSMA7 and LAMA5), methylation and chromatin remodeling (ASXL1, AHCY and C20orf20), as well as transcription regulation (TCEA2) $(16,17)$. Amplification of chromosome 20q deregulates several specific cancer-associated signaling pathways, including the MAPK and p53 signaling pathways (13).

Long non-coding RNAs (lncRNAs), a type of non-coding RNA, serve vital roles in the regulation of various biological processes, including transcription, intracellular trafficking, chromosome remodeling, cell proliferation, metastasis and migration (18-20). Genome-wide association studies of tumor samples have revealed that numerous lncRNAs associated with the processes of tumorigenesis and metastasis become dysregulated with expression alteration and mutations (21-23). Multiple lncRNAs have been considered as either tumor suppressors or oncogenes according to their genome-wide expression patterns and tissue-specific expression characteristics $(21,23)$. Furthermore, IncRNAs have been suggested as novel biomarkers and therapeutic targets for bladder (18), prostate (18), gastric $(19,20)$, pancreatic (24) and breast cancer (25). 
Accumulating studies have indicated that lncRNAs, such as TERRA, HOXA11-AS, AGAP2-AS1, HOTAIR, CCAT1, MALAT1 and Xist, are dysregulated in gastric cancer (GC) (21,26-30). Investigating lncRNAs amplified on 20q may provide novel insights into GC diagnosis and targeted therapy. Therefore, the present study aimed to evaluate lncRNAs on amplified chromosome 20q13.33 in GC tissues and cell lines.

\section{Materials and methods}

GC tissue specimens and cell lines. A total of 60 paired samples of fresh frozen GC tissues and corresponding adjacent non-tumor tissue samples ( $>5 \mathrm{~cm}$ away from tumor) were obtained from The Third Affiliated Hospital of Harbin Medical University (Harbin, China) between March 2006 and March 2008. All cases were reviewed by pathologists and histologically confirmed as GC. Patients were not subjected to local or systemic treatment prior to the procedure. The age range of the 60 patients (46 male and 14 female) was 40-76 years, with a median age of 57 years. The study was approved by the Ethics Committee of Southeast University affiliated to Zhongda Hospital (Nanjing, China).

The normal gastric epithelial GES-1 cell line and the GC AGS, MKN-45 and MKN-74 cell lines were purchased from The Cell Bank of Type Culture Collection of The Chinese Academy of Sciences. The cells were cultured in RPMI-1640 medium containing 10\% FBS (both Gibco; Thermo Fisher Scientific, Inc.) and $1 \%$ penicillin/streptomycin at $37^{\circ} \mathrm{C}$ in a humidified atmosphere with $5 \% \mathrm{CO}_{2}$. The culture medium was changed every 1-2 days and subcultured when the cell confluence reached $80-90 \%$.

$R N A$ extraction and reverse transcription-quantitative $P C R$ $(R T-q P C R)$. Total RNA was extracted from the fresh GC tissues and cell lines with TRIzol ${ }^{\circledR}$ reagent (Thermo Fisher Scientific, Inc.) according to the manufacturer's instructions. cDNA was synthesized using PrimeScript ${ }^{\circledR}$ RT Reagent kit (Takara Bio, Inc.) under standard conditions according to the manufacturer's instructions. RT-qPCR was performed to determine the expression levels of specific genes using a SYBR Premix Ex Taq kit (Takara Bio, Inc.), and $\beta$-actin was used as the internal control for normalization of the data. The thermocycling conditions for amplification were as follows: $95^{\circ} \mathrm{C}$ for $5 \mathrm{~min}$, followed by 40 cycles at $95^{\circ} \mathrm{C}$ for $30 \mathrm{sec}, 60^{\circ} \mathrm{C}$ for $30 \mathrm{sec}$ and $72^{\circ} \mathrm{C}$ for $30 \mathrm{sec}$, and a final step at $72^{\circ} \mathrm{C}$ for $10 \mathrm{~min}$. All the experiments were performed on a StepOne Plus system (Applied Biosystems; Thermo Fisher Scientific, Inc.), and the sequences of the primers for RT-qPCR are shown in Table SI. Relative gene expression compared with its control was assessed using the $2^{-\Delta \Delta \mathrm{Cq}}$ method (31). Each sample was analyzed in triplicate.

Cell transfection. For in vitro assays, the small interfering (si)RNA sequences and non-targeting negative controls (NCs) were synthesized by Shanghai GenePharma Co., Ltd., and were as follows: si-LINC00659-1, 5'-GGGACUUGGAUG CUUAACATT-3'; si-LINC00659-2, 5'-GCCGUGCUCUGG AUAUAUATT-3'; and si-NC, 5'-UUCUCCGAACGUGUC ACGUTT-3'. Short hairpin (sh)RNA sequences were designed according to the aforementioned siRNA sequences. Human embryonic kidney 293T cells were used for lentiviral packing (second-generation packaging system). 293T cells were provided by Shanghai GeneChem Co., Ltd., and originally purchased from the American Type Culture Collection. The cells were cultured in 10-cm dishes for 2-3 days until they reached $90-95 \%$ confluency. The recombinant virus plasmid GV112 $(20 \mu \mathrm{g})$, which encoded sh-LINC00659, and the control, together with packaging plasmids pHelper $1.0(15 \mu \mathrm{g})$ and pHelper $2.0(10 \mu \mathrm{g})$, were co-transfected into $293 \mathrm{~T}$ cells using Lipofectamine ${ }^{\circledR} 2000$ (all from Shanghai GeneChem Co., Ltd.). After $48 \mathrm{~h}$ of transduction, the lentiviral particles contained in the supernatant of $293 \mathrm{~T}$ cells were harvested and then concentrated by passing through a $0.45-\mu \mathrm{m}$ filter.

GC cells $\left(1 \times 10^{5}\right.$ cells/well) were seeded on a 24 -well plate, and cell transfection was performed when the cell number reached $\sim 2 \times 10^{5}$ cells/well. Fresh medium containing $6 \mu \mathrm{g} / \mathrm{ml}$ polybrene was added for transfection at a multiplicity of infection of 10 with control or sh-LINC00659 lentiviruses (Shanghai GeneChem Co., Ltd.) at $37^{\circ} \mathrm{C}$ after the cells had been washed 3 times with PBS. After $8 \mathrm{~h}$ of incubation at $37^{\circ} \mathrm{C}$, fresh medium was added to the cells, and puromycin $(2 \mu \mathrm{g} / \mathrm{ml})$ was added to screen the transfected cells on the 3rd day of transfection. After 7 days, the cells were used for subsequent experimentation.

Cell Counting Kit (CCK)-8 assay. The infected cells were suspended and seeded in a 96 -well plate at a density of 2,000 cell/well. After $0,24,48,72$ and $96 \mathrm{~h}$ of incubation at $37^{\circ} \mathrm{C}$, the culture medium was replaced with $100 \mu \mathrm{lCCK}-8$ reagent (Dojindo Molecular Technologies, Inc.). After $4 \mathrm{~h}$ of incubation, the cell proliferative ability was determined by measuring the optical density at $450 \mathrm{~nm}$.

Wound healing assay. The human GC AGS and MKN-74 cell lines, which were stably transfected with sh-LINC00659, were seeded in 6-well plates and reached a density of $~ 90 \%$ after one day. A 200- $\mu$ l pipette tip was used to scratch the cell culture surface. The cells were washed three times with PBS and were then cultured in serum-free medium. The cells were cultured at $37^{\circ} \mathrm{C}$ in a humidified atmosphere with $5 \% \mathrm{CO}_{2}$. Wound healing was recorded at 0 and $24 \mathrm{~h}$ using an inverted light microscope (Olympus Corporation; magnification, x200).

Cell migration and invasion assay. The cell invasive and migratory potential was measured using Transwell chambers (EMD Millipore) containing a polycarbonate membrane with $8.0-\mu \mathrm{m}$ pores. The human GC AGS and MKN-74 cell lines were stably transfected with sh-LINC00659 using a lentivirus system, as aforementioned. After $48 \mathrm{~h}$ of transfection, $1.5 \times 10^{4}$ cells in serum-free medium were placed into the upper chamber of an insert for migration, and the cells were allowed to migrate for $18 \mathrm{~h}$ at $37^{\circ} \mathrm{C}$. For the invasion assay, $2 \times 10^{4}$ cells in serum-free medium were seeded in Matrigel-coated (Sigma-Aldrich; Merck $\mathrm{KGaA}$ ) inserts and allowed to invade for $36 \mathrm{~h}$ at $37^{\circ} \mathrm{C}$. In both assays, the lower Transwell chamber was filled with medium supplemented with $10 \%$ FBS. After incubation, the cells that had migrated or invaded through the membrane were stained with methanol and $0.1 \%$ crystal violet for $30 \mathrm{~min}$ at room temperature, imaged and counted using an IX71 inverted light microscope (Olympus Corporation; magnification, x200). 
The Cancer Genome Atlas (TCGA) data analysis. Processed TCGA expression data including tumor $(\mathrm{n}=408)$ and normal samples $(n=36)$ of patients with $\mathrm{GC}$ were downloaded from TCGA database (http://cancergenome.nih.gov/), and the upper quantile normalized fragments per kilobase per million values were collected and converted into $\log 2$ data form. The data were used to analyze the survival of patients with GC based on lncRNA expression with Kaplan-Meier survival curves. The samples were first divided into two groups (high and low LINC00659 groups) according to the median expression levels (FPKM value, 37) of LINC00659. The 5 -year survival analysis was performed using the survival package (https://cran.r-project.org/web/packages/survival) in the R software.

Enrichment analysis. To explore the potential downstream pathways of LINC00659, LINC00659 expression was categorized into two groups of high and low expression, according to the median value (FPKM value, 37) of LINC00659 expression. Gene set enrichment analysis (GSEA) 2-2.2.3 (JAVA version) was downloaded from the GSEA website (http://www. gsea-msigdb.org/gsea/downloads.jsp). Subsequently, the downloaded dataset was imported using the GSEA software. Gene sets associated with biological signal transduction were identified on the Molecular Signatures Database (MSigDB; http://software.broadinstitute.org/gsea/msigdb). The simulation was repeated 1,000 times for each analysis, according to the default weighted enrichment statistical method. Gene sets with a false discovery rate $<0.25$ and $\mathrm{P}<0.05$ were selected.

Target gene prediction. The correlation between the co-expression of LINC00659 and all protein coding genes (PCGs) was determined by calculating the Pearson correlation coefficients and $\mathrm{z}$ test. The PCGs positively or negatively correlated with LINC00659 were considered as LINC00659-associated PCGs (IPearson correlation coefficient $\mid>0.4$ and $\mathrm{P}<0.01$ ).

Statistical analysis. All experiments were performed independently at least three times and data are presented as the mean \pm SD. Statistical analyses were performed using SPSS 18.0 software (SPSS, Inc.). Comparisons between two groups were made using the independent sample t-test; unpaired Student's t-test was used to analyze the differences between two groups, and paired Student's t-test was used to compare the paired tissue samples. Comparisons among $>2$ groups were made using ANOVA followed by Fisher's LSD post-hoc test (for 3 groups) or Dunnett's post-hoc test (for $>3$ groups). Fisher's exact test or $\chi^{2}$ test was used to analyze the association between LINC00659 expression and clinicopathological parameters. Survival curves were plotted using the Kaplan-Meier method, and the difference in OS survival rates was assessed using the log-rank test. $\mathrm{P}<0.05$ was considered to indicate a statistically significant difference.

\section{Results}

LINC00659 expression is upregulated and associated with a poor prognosis in patients with GC included in TCGA database. Alteration of chromosomal duplication and gene amplification are crucial for functional gain and overexpression of oncogenes. Based on the CGH analysis, several chromosomal aberrations, especially recurrent gain and amplification of the long arm of chromosome 20 (20q13.33), have been observed in numerous types of cancer, including GC (12-17). In order to explore the contribution of lncRNAs located on amplified 20q13.33 (Fig. 1A), TCGA database was screened, and the expression levels of IncRNAs in $\mathrm{GC}$ were analyzed. The expression levels of four lncRNAs, namely RTEL1-TNFRSF6B, SLCO4A1-AS1, ZBTB46-AS1 and LINC00659, were found to be significantly upregulated in gastric tumor tissues compared with in non-tumor tissues (Fig. 1B). Subsequently, Kaplan-Meier analysis using a log-rank test was performed to investigate whether the expression levels of these four lncRNAs were associated with the survival of patients with GC. The results demonstrated that patients with a high level of LINC00659 had a significantly poorer OS rate $(\mathrm{P}=0.04)$ compared with patients with a low level of LINC00659 expression (Fig. 1C). However, the other three IncRNAs, RTEL-TNFRSF6B, SLCO4A1-AS1 and ZBTB46-AS1, were not associated with OS rate or tumor stage (Figs. S1 and S2). Additionally, the expression levels of LINC00659 were significantly increased in patients with advanced GC (stage IV) compared with in those with stages I-III (Fig. 1D). These data indicated that patients with GC with increased levels of LINC00659 expression may be prone to progress to a more advanced stage of the disease.

LINC00659 expression is upregulated in GC clinical tissues and cell lines. The relative expression levels of LINC00659 were detected in 60 paired GC and adjacent non-cancerous tissues via RT-qPCR. LINC00659 expression was significantly upregulated in the cancerous tissues compared with in their non-cancerous counterparts (Fig. 2A). Furthermore, the association of LINC00659 expression with clinical features of GC was examined, revealing that higher expression levels of LINC00659 were associated with differentiation and vessel invasion; however, no association was observed with sex, age, tumor size, lymph node metastasis and Helicobacter pylori infection (Table SII). Additionally, the expression levels of LINC00659 were higher in cancer tissues than in adjacent non-cancerous tissues in patients with lymph node metastasis (Fig. 2B). Subsequently, LINC00659 expression was detected in three GC cell lines (AGS, MKN74 and MKN-45) and the normal gastric epithelial GES-1 cell line via RT-qPCR. As presented in Fig. 2C, all three GC cell lines exhibited significantly higher expression levels of LINC00659 compared with the normal gastric epithelial cell line. Furthermore, LINC00659 was more highly expressed in the copy number-amplified group than in the normal copy number group $(\mathrm{P}<0.01$; Fig. 2D). This finding indicated that the number of amplifications of LINC00659 may be closely associated with its expression. Overall, these results indicated that upregulation of LINC00659 expression may have important roles in GC development and progression.

Function of LINC00659 in GC cells. To explore the biological function of LINC00659, GSEA analysis based on the mRNA expression levels of LINC00659 in GC was performed using TCGA data. Enrichment plots of gene expression signatures illustrated that epithelial cell migration and 


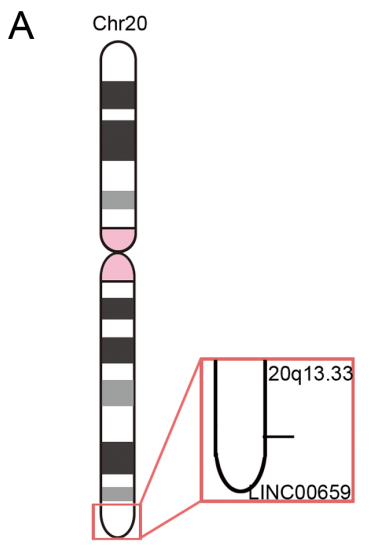

C

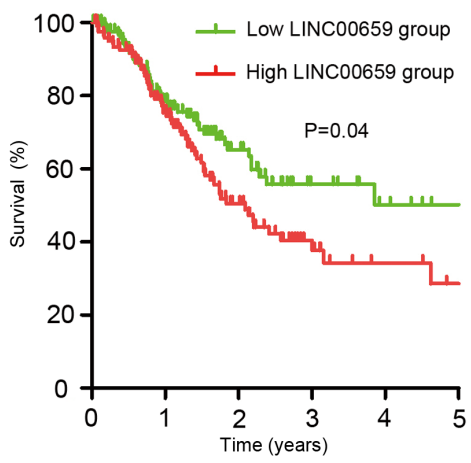

B

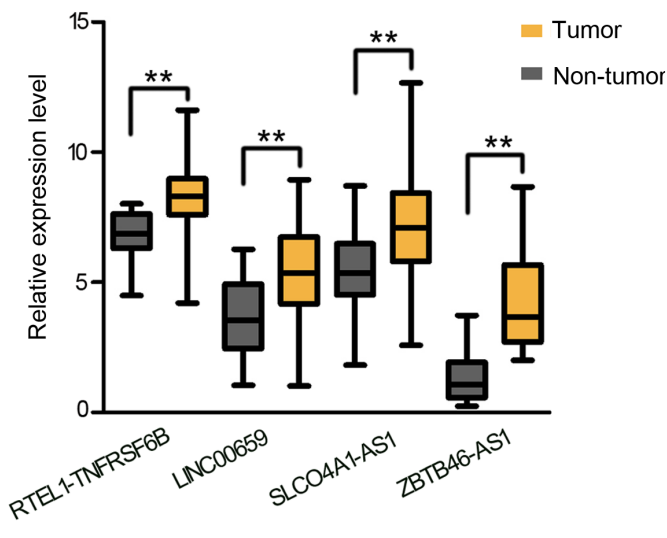

$\mathrm{D}$

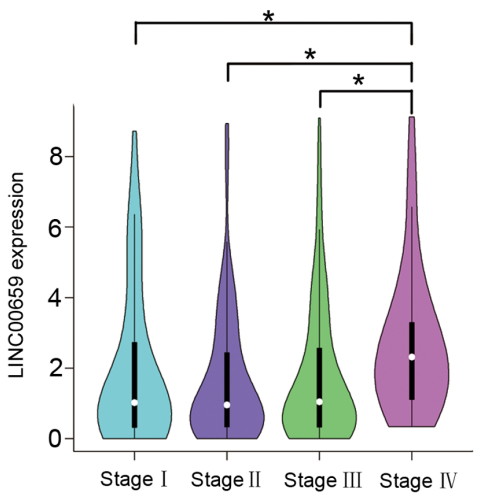

Figure 1. Increased LINC00659 expression is associated with a poor prognosis in patients with GC. (A) LINC00659 is located on Chr20q13.33. (B) Expression levels of four lncRNAs located on Chr20q13.33 were upregulated in GC tissues compared with in their corresponding adjacent non-tumor tissues by The Cancer Genome Atlas database analysis. ${ }^{* *} \mathrm{P}<0.01$ (unpaired Student's t-test). (C) Survival time of patients with GC (categorized as high and low LINC00659 expression groups) was analyzed using the log-rank test. (D) LINC00659 expression was significantly increased in the advanced stage of GC. "P<0.05 (ANOVA with Dunnett's post-hoc test). GC, gastric cancer; Chr, chromosome.

A

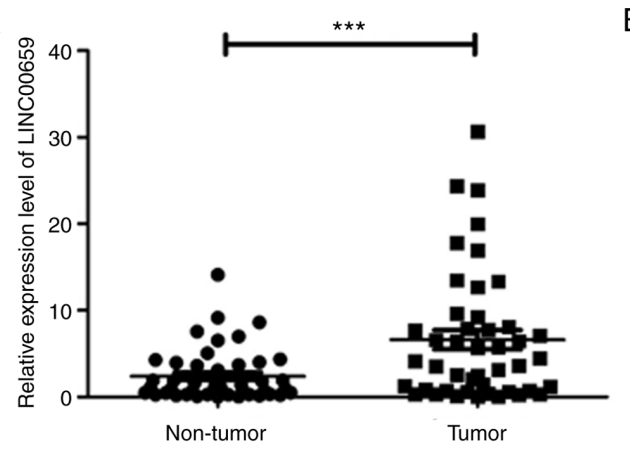

C

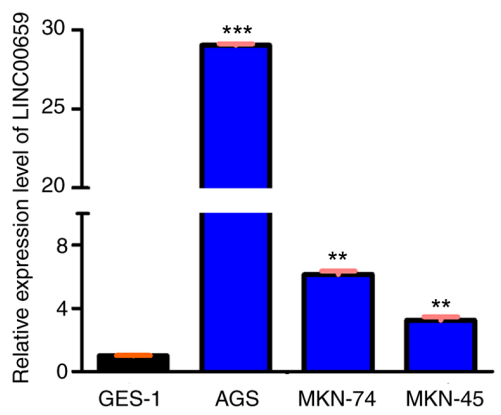

$B$

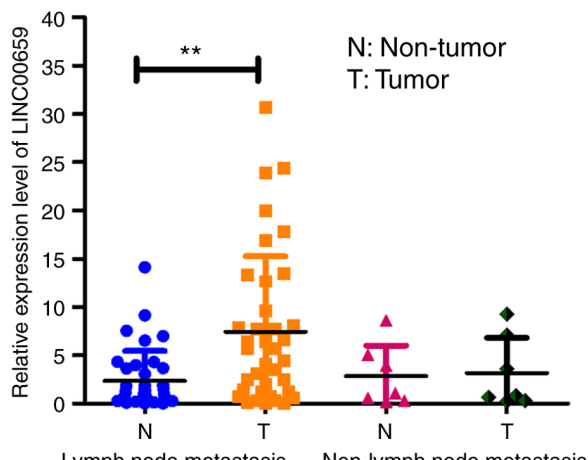

Lymph node metastasis Non-lymph node metastasis

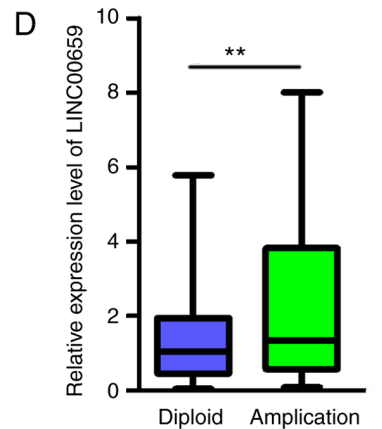

Figure 2. Increased LINC00659 expression is associated with lymph node metastasis in GC. (A) LINC00659 expression was measured by RT-qPCR in 60 paired gastric cancerous tissues and non-cancerous epithelial tissues. ${ }^{* * * *} \mathrm{P}<0.001$ (paired Student's t-test). (B) LINC00659 expression was upregulated in GC tumor tissues with lymph node metastasis compared with in non-tumor tissues. ${ }^{* *} \mathrm{P}<0.01$ (paired Student's t-test). (C) LINC00659 expression was analyzed by RT-qPCR in GC cell lines (AGS, MKN-74 and MKN-45) compared with the normal gastric epithelium cell line (GES-1). ${ }^{* *} \mathrm{P}<0.01$ and ${ }^{* * *} \mathrm{P}<0.001(\mathrm{ANOVA}$ followed by Dunnett's post-hoc test). (D) LINC00659 expression was compared between the diploid and amplification groups of patients with GC. ${ }^{* *} \mathrm{P}<0.01$ (unpaired Student's t-test). GC, gastric cancer; RT-qPCR, reverse transcription-quantitative PCR. 
A

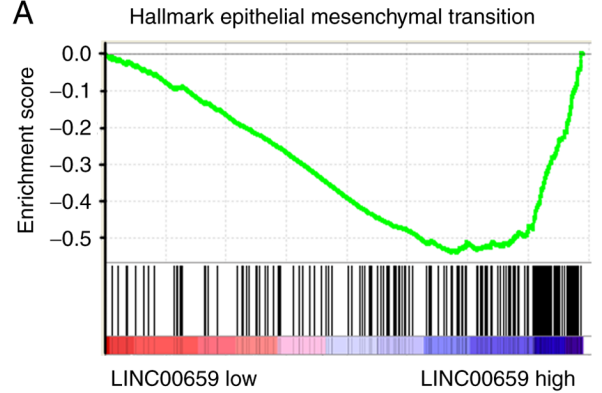

B

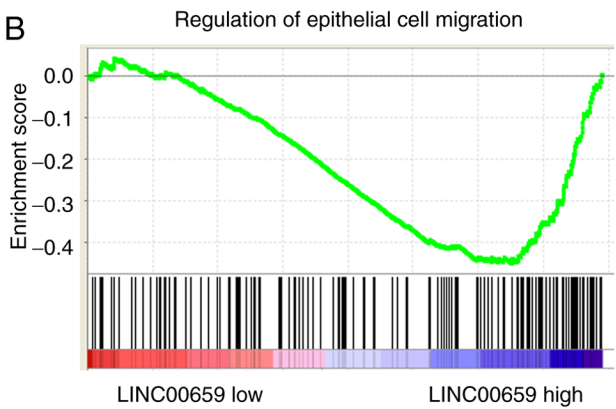

C

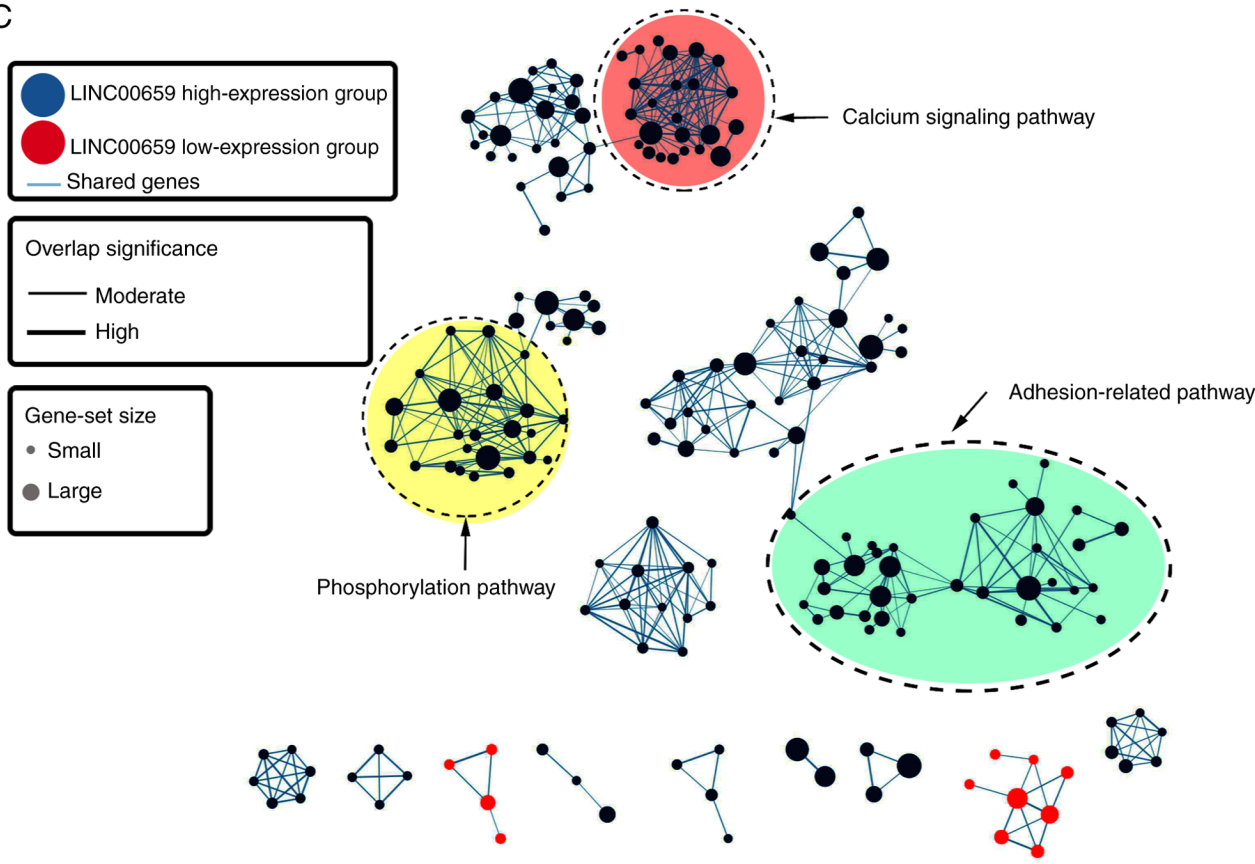

Figure 3. Enrichment plots of gene expression signatures indicating the potential function of LINC00659. According to LINC00659 expression, enrichment plots of gene expression signatures were focused on (A) epithelial-mesenchymal transition and (B) epithelial cell migration. The bar-code plot indicates the positions of genes in each gene set; the colors red and blue represent low and high LINC00659 expression, respectively. (C) GSEA comparison between patients in the high (blue) and low (red) LINC00659 expression groups. Distinct pathways and biological processes were illustrated between the two patient populations. The enrichment map was used for visualization of the GSEA results (FDR $<0.25$ and $\mathrm{P}<0.05$ ). Nodes represent enriched gene sets, which were grouped and annotated by their similarity according to the related gene sets. Enrichment results were mapped as a network of gene sets (nodes), and the node size was proportional to the total number of genes within each gene set. GSEA, gene set enrichment analysis.

epithelial-mesenchymal transition pathways were associated with the expression levels of LINC00659 (Fig. 3A and B). With further analysis, the potential function of LINC00659 was aggregated at a few functional clusters, including calcium signaling pathway, phosphorylation pathway and adhesion-related pathway (Fig. 3C). These data implied that the function of LINC00659 may be associated with cell migration and invasion involved in GC metastasis.

LINC00659 has no effect on cell proliferation according to CCK-8 assay. To assess the effects of LINC00659 expression on GC cells, sh-LINC00659 or sh-NC were used to infect AGS and MKN-74 cells, which exhibited higher LINC00659 expression. The efficiency of infection was confirmed by RT-qPCR, and a significant decrease in LINC00659 expression was observed following transfection with both sh-LINC00659 molecules (Fig. 4A), with sh-LINC00659-2 being chosen for subsequent experiments. The CKK-8 assay revealed that LINC00659 inhibition by sh-LINC00659-2 did not affect the proliferation of AGS and MKN-74 cells (Fig. S3).
LINC00659 promotes GC cell migration and invasion in vitro. In order to clarify the function of LINC00659 in terms of cell migration and invasion, the effects of LINC00659-knockdown on cell migration and invasion were assessed. The results of the wound-healing assays revealed that the migratory ability of AGS and MKN-74 cells was decreased in the LINC00659-knockdown group compared with in the NC group (Fig. 4B). Transwell migration assays displayed that LINC00659-knockdown significantly suppressed the migratory capabilities of both AGS and MKN-74 cancer cells compared with the control group (Fig. 4C and E). Moreover, GC cells with LINC00659-knockdown exhibited significantly weaker abilities to invade through Matrigel compared with the control group (Fig. 4D and F). These results indicated that knocking down LINC00659 led to a clear suppression of the migratory and invasive abilities of GC cells.

IQ motif-containing GTPase activating protein 3 (IQGAP3) and matrix metalloproteinase 15 (MMP15) are potential downstream targets involved in LINC00659-induced tumor metastasis. To investigate the underlying mechanism of 
A
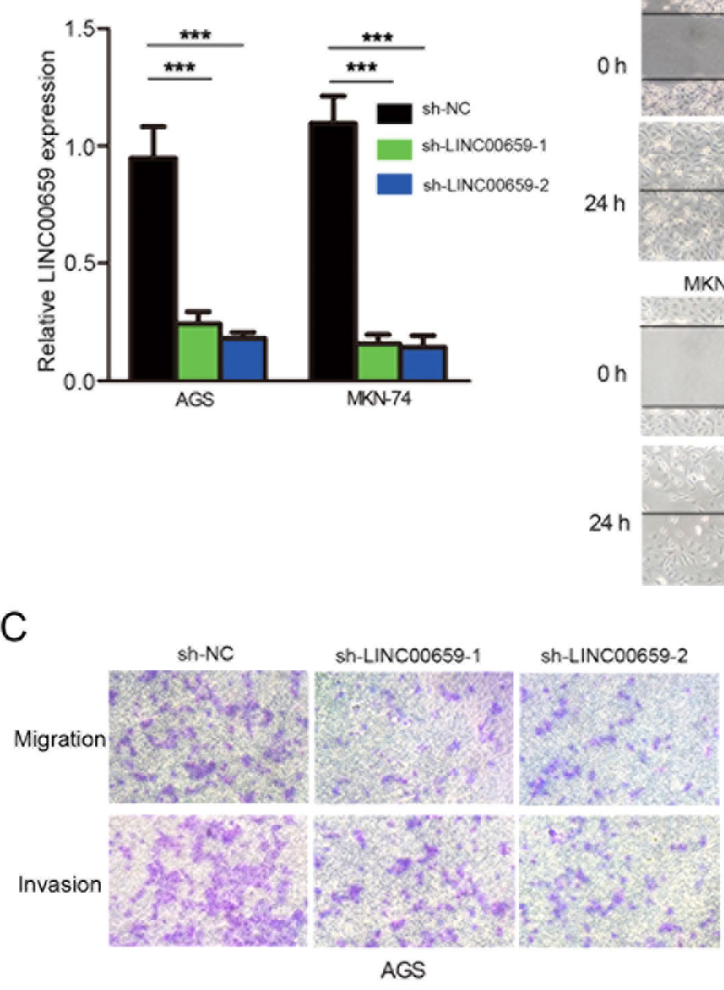

E

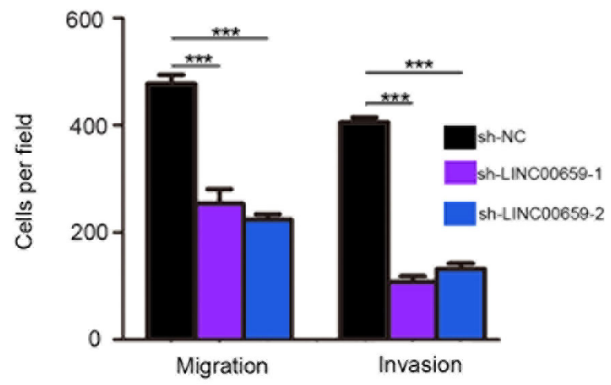

B
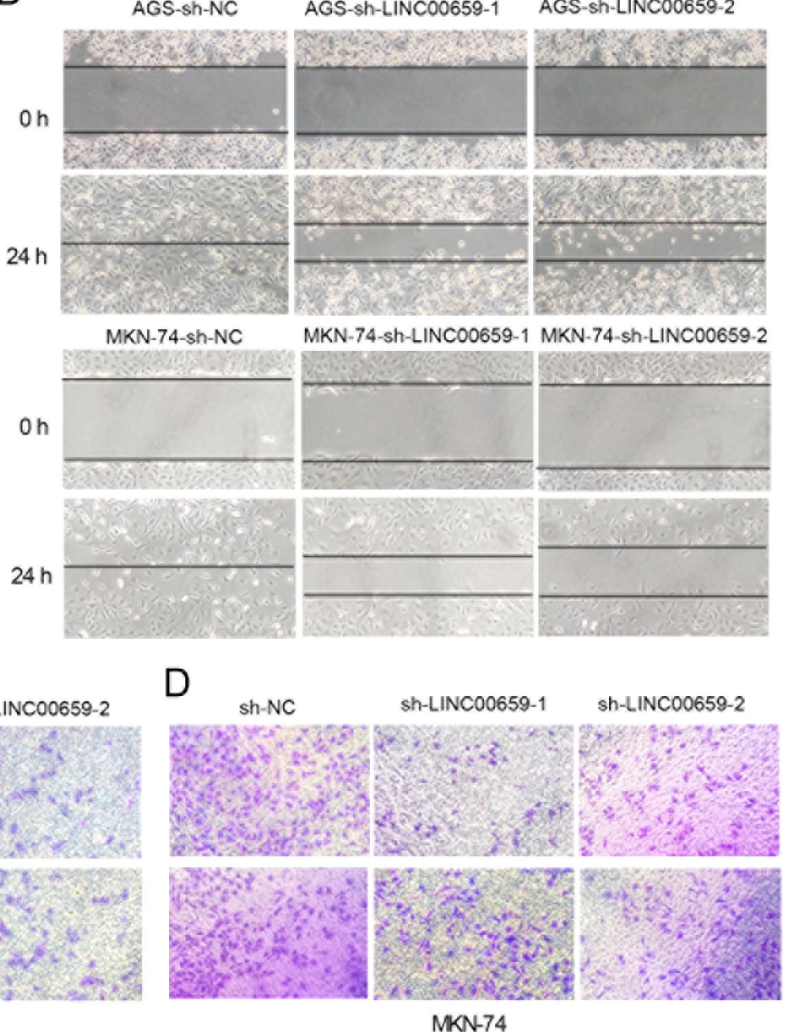

$\mathrm{F}$

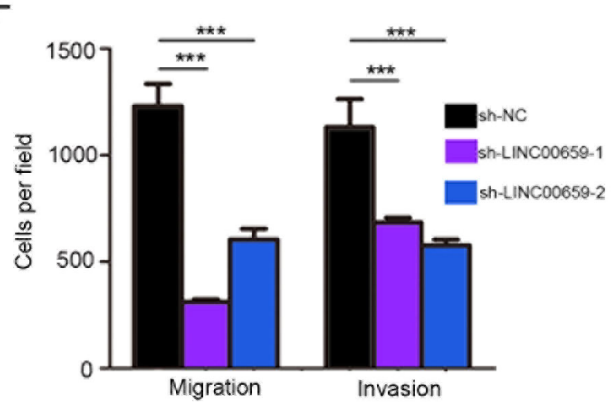

Figure 4. Functions of LINC00659 on GC cell migration and invasion. (A) Efficiency of sh-LINC00659, which was used to knock down LINC00659 expression, was detected in AGS and MKN-74 cells. (B) Wound healing assay revealing the migratory abilities of AGS and MKN-74 cells after knocking down LINC00659 with sh-LINC00659 (magnification, x200). After knocking down LINC00659 expression with sh-LINC00659, cell migration and invasion assays were performed in (C) AGS and (D) MKN-74 cells (magnification, x200). Bar charts show the quantification of the data in (E) AGS and (F) MKN-74 cells, showing the mean number of migrating/invading cells $( \pm \mathrm{SD})$ per microscopic field from triplicate samples. Statistical analysis was performed using ANOVA with Fisher's LSD post-hoc test. ${ }^{* * *} \mathrm{P}<0.001$. sh-NC, short hairpin RNA negative control.

LINC00659 in cell migration and invasion, the correlation between the expression levels of LINC00659 and all PCGs was examined using two-sided Pearson correlation coefficients and z-test. The PCGs positively or negatively correlated with LINC00659 were considered as LINC00659-associated PCGs (IPearson correlation coefficientl $>0.4$ and $\mathrm{P}<0.01$ ). The expression levels of eight genes, namely IQGAP3, MMP15, integrin subunit $\alpha 6$ (ITGA6), G protein-coupled receptor class C group 5 member A (GPRC5A), hematological and neurological expressed 1 (HN1), keratin 8 (KRT8), engulfment and cell motility 3 (ELMO3), and TNF receptor associated factor 4 (TRAF4), were found to be positively correlated with LINC00659 expression (Fig. 5A-H). To determine whether these eight genes were downstream targets of LINC00659, their expression patterns were investigated in GC cells where LINC00659 expression had been knocked down by sh-LINC00659-2. As shown in Fig. 6A and B, the expression levels of IQGAP3 and MMP15 were significantly downregulated in LINC00659-knockdown GC cells, and ITGA6 expression was significantly downregulated only in AGS cells. GPRC5A, HN1, KRT8, ELMO3 and TRAF4 exhibited no change in expression when LINC00659 was knocked down in AGS and MKN-74 cells (Fig. 6C). These results suggested that LINC00659 may be associated with metastasis via IQGAP3 and MMP15 as its downstream targets.

\section{Discussion}

An increasing number of studies has revealed that lncRNAs serve a critical role in cancer $(22,32,33)$, and although a 

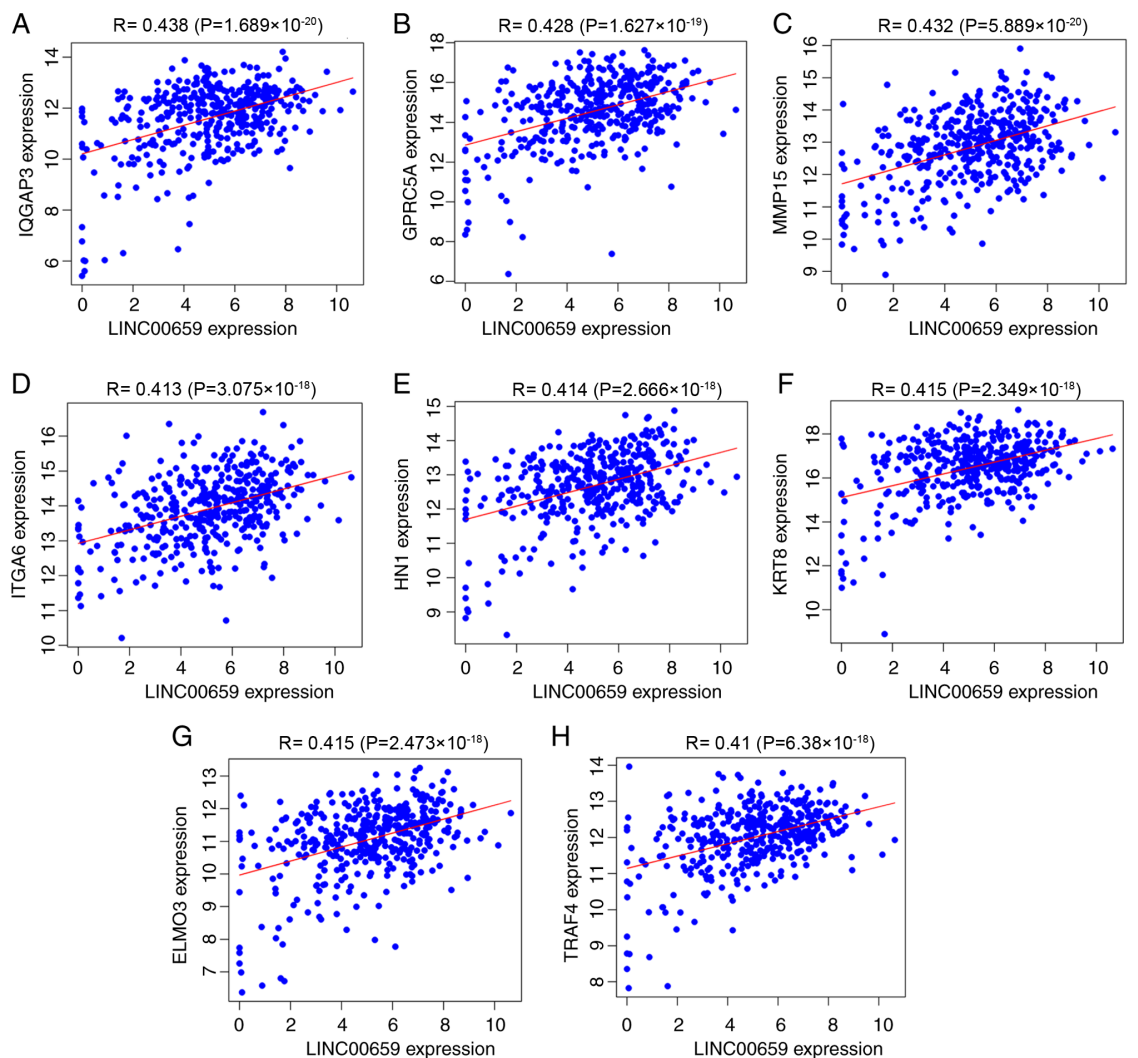

Figure 5. Pearson correlation analysis of the expression levels of eight protein-coding genes co-expressed with LINC00659 expression in The Cancer Genome Atlas database. Correlation of LINC00659 expression with (A) IQGAP3, (B) GPRC5A, (C) MMP15, (D) ITGA6, (E), HN1, (F) KRT8, (G) ELMO3 and (H) TRAF4. IQGAP3, IQ motif-containing GTPase activating protein 3; MMP15, matrix metalloproteinase 15; ITGA6, integrin subunit $\alpha 6$; GPRC5A, G protein-coupled receptor class C group 5 member A; HN1, hematological and neurological expressed 1; KRT8, keratin 8; ELMO3, engulfment and cell motility 3; TRAF4, TNF receptor associated factor 4.
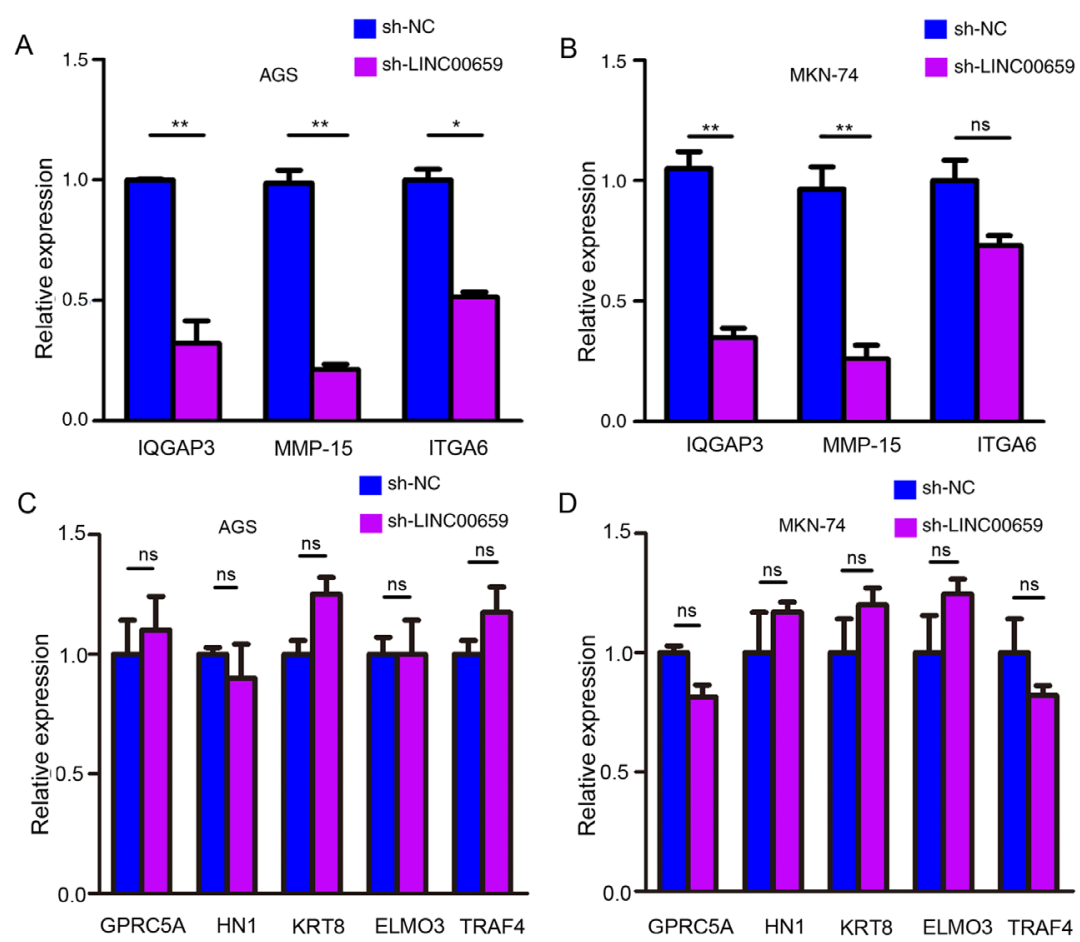

Figure 6. IQGAP3 and MMP-15 are downstream targets of LINC00659. Relative expression levels of potential target genes (IQGAP3, MMP-15 and ITGA6) detected by reverse transcription-quantitative PCR in (A) AGS and (B) MKN-74 cells following transfection with sh-LINC00659. (C) Relative expression levels of GPRC5A, HN1, KRT8, ELMO3 and TRAF4 following LINC00659-knockdown in AGS. (D) Relative expression levels of GPRC5A, HN1, KRT8, ELMO3 and TRAF4 following LINC00659-knockdown in MKN-74. "P<0.05; * P<0.01 (Student's unpaired t-test). MMP15, matrix metalloproteinase 15; IQGAP3, IQ motif-containing GTPase activating protein 3; ITGA6, integrin subunit $\alpha 6$; GPRC5A, G protein-coupled receptor class $\mathrm{C}$ group 5 member A; HN1, hematological and neurological expressed 1; KRT8, keratin 8; ELMO3, engulfment and cell motility 3; TRAF4, TNF receptor associated factor 4; sh-NC, short hairpin RNA negative control; ns, not significant. 
large number of lncRNAs have been identified in the human genome, only a small number of them have been experimentally validated and functionally annotated in GC (34). The initiation and progression of GC involve deregulation of numerous lncRNAs (35). Some lncRNA functions have been uncovered, so that they have become potential biomarkers and therapeutic targets for the prognosis and treatment of GC (36). For example, the IncRNA HOXA11-AS promotes GC cell proliferation and invasion via serine/arginine-rich splicing factor 1, and may function as a putative marker in GC (37). Additionally, lncRNA antisense non-coding RNA in the INK4 locus may have potential as a biomarker and therapeutic target for GC prognosis and treatment (38). LINC00337, MNX1-AS1 and MALAT1 have been shown to promote proliferation, migration and invasion of GC cells (39-41). Genomic amplifications are hallmarks of cancer, and investigation of non-coding elements, such as lncRNAs, localized on amplicons is often employed in research to delineate their roles in malignant transformation (37-40,42). A frequent expansion on chromosome 20 by CGH has often been demonstrated (43), and the chromosome has numerous GC susceptibility loci in 20q13.33 (44,45). The present study identified four lncRNAs, namely RTEL1-TNFRSF6B, SLCO4A1-AS1, ZBTB46-AS1 and LINC00659, in this amplified 20q13.33 region, which were differentially expressed in GC tissues and para-cancerous tissues; however, only LINC00659 was associated with the prognosis of patients with GC. Additionally, genetic alterations of LINC00659 amplification were significantly associated with higher expression levels of LINC00659.

A previous study has revealed that LINC00659 is a novel oncogenic lncRNA involved in colon cancer cell proliferation via modulating the cell cycle (46). LINC00659-knockdown significantly suppresses colon cancer cell proliferation by impairing cell cycle progression and suppressing PI3K-AKT signaling (38). However, to the best of our knowledge, the expression pattern and functional significance of LINC00659 in GC cell migration and invasion have remained elusive. In the present study, LINC00659 expression was shown to be upregulated in GC tissues. The expression levels of LINC00659 were associated with GC stage, and LINC00659 expression was significantly increased in patients with advanced GC. Additionally, the expression levels of LINC00659 were higher in cancer tissues than in adjacent non-cancerous tissues in patients with lymph node metastasis. After knocking down LINC00659, the AGS and MKN-74GC cell lines displayed significantly attenuated migratory and invasive abilities, although LINC00659-knockdown had no influence on cell proliferation. IQGAP3 and MMP15 were identified as potential downstream targets of LINC00659, with crucial roles in GC metastasis. However, a limitation of the present study is that the function of these two downstream targets was not further analyzed in in vitro and in vivo experiments. Wound healing, Transwell and tail vein injection assays to observe the migration and invasion of GC cells following IQGAP3- and MMP15-knockdown may provide more robust evidence for the current findings.

In summary, the present study has identified a novel lncRNA associated with a poor prognosis in patients with GC. LINC00659 was identified as an oncogenic regulator that promoted GC cell migration and invasion. The current findings suggested that IQGAP3 and MMP15 may be putative targets for LINC00659, although the mechanism requires further investigation. The present findings indicated that LINC00659 may be an important molecular marker in GC tumorigenesis, and may have the potential to be a novel promising prognostic and therapeutic maker for patients with GC.

\section{Acknowledgements}

Not applicable.

\section{Funding}

The present study was supported by the National Natural Science Foundation of China (grant nos. 81972664 and 81672414).

\section{Availability of data and materials}

The datasets used and/or analyzed during the current study are available from the corresponding author on reasonable request.

\section{Authors' contributions}

PG, YL, KZ and YX performed the experiments, analyzed the data and wrote the manuscript. ML, XS, SY and YM performed the experiments and analyzed the data. HF designed the experiments, supervised the project and revised the manuscript. KZ, YL and SY confirm the authenticity of all the raw data. All authors read and approved the final manuscript.

\section{Ethics approval and consent to participate}

The present study was approved by the Research Ethics Committee of Southeast University affiliated to Zhongda Hospital. Verbal informed consent was provided by all patients included in the study.

\section{Patient consent for publication}

Not applicable.

\section{Competing interests}

The authors declare that they have no competing interests.

\section{References}

1. Bray F, Ferlay J, Soerjomataram I, Siegel RL, Torre LA and Jemal A: Global cancer statistics 2018: GLOBOCAN estimates of incidence and mortality worldwide for 36 cancers in 185 countries. CA Cancer J Clin 68: 394-424, 2018.

2. Karimi P, Islami F, Anandasabapathy S, Freedman ND and Kamangar F: Gastric cancer: Descriptive epidemiology, risk factors, screening, and prevention. Cancer Epidemiol Biomarkers Prev 23: 700-713, 2014.

3. Solomon E, Borrow J and Goddard AD: Chromosome aberrations and cancer. Science 254: 1153-1160, 1991.

4. Takada H, Imoto I, Tsuda H, Sonoda I, Ichikura T, Mochizuki H, Okanoue T and Inazawa J: Screening of DNA copy-number aberrations in gastric cancer cell lines by array-based comparative genomic hybridization. Cancer Sci 96: 100-110, 2005. 
5. Hidaka S, Yasutake T, Kondo M, Takeshita H, Yano H, Haseba M, Tsuji T, Sawai T, Nakagoe T and Tagawa Y: Frequent gains of $20 \mathrm{q}$ and losses of $18 \mathrm{q}$ are associated with lymph node metastasis in intestinal-type gastric cancer. Anticancer Res 23: 3353-3357, 2003.

6. Oga A, Kong G, Ishii Y, Izumi H, Park CY and Sasaki K: Preferential loss of 5q14-21 in intestinal-type gastric cancer with DNA aneuploidy. Cytometry 46: 57-62, 2001.

7. Kokkola A, Monni O, Puolakkainen P, Larramendy ML, Victorzon M, Nordling S, Haapiainen R, Kivilaakso E and Knuutila S: 17q12-21 amplicon, a novel recurrent genetic change in intestinal type of gastric carcinoma: A comparative genomic hybridization study. Genes Chromosomes Cancer 20: 38-43, 1997.

8. Wu MS, Chang MC, Huang SP, Tseng CC, Sheu JC, Lin YW, Shun CT, Lin MT and Lin JT: Correlation of histologic subtypes and replication error phenotype with comparative genomic hybridization in gastric cancer. Genes Chromosomes Cancer 30 : $80-86,2001$.

9. Kwon MJ, Kim RN, Song K, Jeon S, Jeong HM, Kim JS, Han J, Hong S, Oh E, Choi JS, et al: Genes co-amplified with ERBB2 or MET as novel potential cancer-promoting genes in gastric cancer. Oncotarget 8: 92209-92226, 2017.

10. Tabach Y, Kogan-Sakin I, Buganim Y, Solomon H, Goldfinger N, Hovland R, Ke XS, Oyan AM, Kalland KH, Rotter V and Domany E: Amplification of the 20q chromosomal arm occurs early in tumorigenic transformation and may initiate cancer. PLoS One 6: e14632, 2011.

11. Garcia-Murillas I, Sharpe R, Pearson A, Campbell J, Natrajan R, Ashworth A and Turner NC: An siRNA screen identifies the GNAS locus as a driver in $20 \mathrm{q}$ amplified breast cancer. Oncogene 33: 2478-2486, 2014.

12. Sana M and Malik HJ: Current and emerging breast cancer biomarkers. J Cancer Res Ther 11: 508-513, 2015.

13. Ptashkin RN, Pagan C, Yaeger R, Middha S, Shia J, O'Rourke KP, Berger MF, Wang L, Cimera R, Wang J, et al: Chromosome 20c amplification defines a subtype of microsatellite stable, left-sided colon cancers with wild-type RAS/RAF and better overall survival. Mol Cancer Res 15: 708-713, 2017.

14. Sillars-Hardebol AH, Carvalho B, Belien JA, de Wit M, Delis-van Diemen PM, Tijssen M, van de Wiel MA, Ponten F, Fijneman RJ and Meijer GA: BCL2L1 has a functional role in colorectal cancer and its protein expression is associated with chromosome 20q gain. J Pathol 226: 442-450, 2012.

15. Hodgson JG, Chin K, Collins C and Gray JW: Genome amplification of chromosome 20 in breast cancer. Breast Cancer Res Treat 78: 337-345, 2003.

16. Snijders AM and Mao JH: Multi-omics approach to infer cancer therapeutic targets on chromosome $20 \mathrm{q}$ across tumor types. Adv Mod Oncol Res 2: 215-223, 2016.

17. Scotto L,Narayan G,Nandula SV,Arias-Pulido H,Subramaniyam S Schneider A, Kaufmann AM, Wright JD, Pothuri B, Mansukhani M and Murty VV: Identification of copy number gain and overexpressed genes on chromosome arm 20q by an integrative genomic approach in cervical cancer: Potential role in progression. Genes Chromosomes Cancer 47: 755-765, 2008.

18. Bolha L, Ravnik-Glavac M and Glavac D: Long noncoding RNAs as biomarkers in cancer. Dis Markers 2017: 7243968, 2017.

19. Zeng S, Xie X, Xiao YF, Tang B, Hu CJ, Wang SM, Wu YY, Dong H, Li BS and Yang SM: Long noncoding RNA LINC00675 enhances phosphorylation of vimentin on Ser83 to suppress gastric cancer progression. Cancer Lett 412: 179-187, 2018.

20. Pan L, Liang W, Fu M, Huang ZH, Li X, Zhang W, Zhang P, Qian H, Jiang PC, Xu WR and Zhang X: Exosomes-mediated transfer of long noncoding RNA ZFAS1 promotes gastric cancer progression. J Cancer Res Clin Oncol 143: 991-1004, 2017.

21. Liu Z, Chen Z, Fan R, Jiang B, Chen X, Chen Q, Nie F, Lu K and Sun M: Over-expressed long noncoding RNA HOXA11-AS promotes cell cycle progression and metastasis in gastric cancer. Mol Cancer 16: 82, 2017.

22. Bhan A, Soleimani M and Mandal SS: Long noncoding RNA and cancer: A new paradigm. Cancer Res 77: 3965-3981, 2017.

23. Forrest ME and Khalil AM: Review: Regulation of the cancer epigenome by long non-coding RNAs. Cancer Lett 407: 106-112, 2017.

24. ENCODEProject Consortium; Birney E,Stamatoyannopoulos JA, Dutta A, Guigo R, Gingeras TR, Margulies EH, Weng Z, Snyder M, Dermitzakis ET, et al: Identification and analysis of functional elements in $1 \%$ of the human genome by the ENCODE pilot project. Nature 447: 799-816, 2007.
25. Chandra Gupta S and Nandan Tripathi Y: Potential of long non-coding RNAs in cancer patients: From biomarkers to therapeutic targets. Int J Cancer 140: 1955-1967, 2017.

26. Qi F, Liu X, Wu H, Yu X, Wei C, Huang X, Ji G, Nie F and Wang K: Long noncoding AGAP2-AS1 is activated by SP1 and promotes cell proliferation and invasion in gastric cancer. J Hematol Oncol 10: 48, 2017.

27. YiRen H, YingCong Y, Sunwu Y, Keqin L, Xiaochun T, Senrui C, Ende C, XiZhou L and Yanfan C: Long noncoding RNA MALAT1 regulates autophagy associated chemoresistance via miR-23b-3p sequestration in gastric cancer. Mol Cancer 16: 174, 2017.

28. Rinn JL, Kertesz M, Wang JK, Squazzo SL, Xu X, Brugmann SA, Goodnough LH, Helms JA, Farnham PJ, Segal E and Chang HY: Functional demarcation of active and silent chromatin domains in human HOX loci by noncoding RNAs. Cell 129: 1311-1323, 2007.

29. Thorvaldsen JL, Duran KL and Bartolomei MS: Deletion of the H19 differentially methylated domain results in loss of imprinted expression of H19 and Igf2. Genes Dev 12: 3693-3702, 1998.

30. Chu C, Zhang QC, da Rocha ST, Flynn RA, Bharadwaj M, Calabrese JM, Magnuson T, Heard E and Chang HY: Systematic discovery of Xist RNA binding proteins. Cell 161: 404-416, 2015.

31. Livak KJ and Schmittgen TD: Analysis of relative gene expression data using real-time quantitative PCR and the 2(-Delta Delta C(T)) method. Methods 25: 402-408, 2001

32. Schmitt AM and Chang HY: Long noncoding RNAs in cancer pathways. Cancer Cell 29: 452-463, 2016.

33. Huarte M: The emerging role of lncRNAs in cancer. Nat Med 21: $1253-1261,2015$

34. Nasrollahzadeh-Khakiani M, Emadi-Baygi M, Schulz WA and Nikpour P: Long noncoding RNAs in gastric cancer carcinogenesis and metastasis. Brief Funct Genomics 16: 129-145, 2017.

35. Sun M, Nie FQ, Wang ZX and De W: Involvement of lncRNA dysregulation in gastric cancer. Histol Histopathol 31: 33-39, 2016.

36. Yuan L, Xu ZY, Ruan SM, Mo S, Qin JJ and Cheng XD: Long non-coding RNAs towards precision medicine in gastric cancer: Early diagnosis, treatment, and drug resistance. Mol Cancer 19: 96, 2020.

37. Liu Y, Zhang YM, Ma FB, Pan SR and Liu BZ: Long noncoding RNA HOXA11-AS promotes gastric cancer cell proliferation and invasion via SRSF1 and functions as a biomarker in gastric cancer. World J Gastroenterol 25: 2763-2775, 2019.

38. Deng W, Zhang Y, Cai J, Zhang J, Liu X, Yin J, Bai Z, Yao H and Zhang Z: LncRNA-ANRIL promotes gastric cancer progression by enhancing NF-kB signaling. Exp Biol Med (Maywood) 244: 953-959, 2019

39. Zhu K, Ren Q and Zhao Y: IncRNA MALAT1 overexpression promotes proliferation, migration and invasion of gastric cancer by activating the PI3K/AKT pathway. Oncol Lett 17: 5335-5342, 2019.

40. Ma JX, Yang YL, He XY, Pan XM, Wang Z and Qian YW: Long noncoding RNA MNX1-AS1 overexpression promotes the invasion and metastasis of gastric cancer through repressing CDKN1A. Eur Rev Med Pharmacol Sci 23: 4756-4762, 2019.

41. Hu B, Wang X and Li L: Long noncoding RNA LINC00337 promote gastric cancer proliferation through repressing $\mathrm{p} 21$ mediated by EZH2. Am J Transl Res 11: 3238-3245, 2019.

42. Li D, Chen Y, Mei H, Jiao W, Song H, Ye L, Fang E, Wang X, Yang $\mathrm{F}$, Huang $\mathrm{K}$, et al: Ets-1 promoter-associated noncoding RNA regulates the NONO/ERG/Ets-1 axis to drive gastric cancer progression. Oncogene 37: 4871-4886, 2018.

43. Nicolet C, Guerin E, Neuville A, Kerckaert JP, Wicker N, Bergmann E, Brigand C, Kedinger M, Gaub MP and Guenot D: Evidence for various $20 \mathrm{q}$ status using allelotyping, CGH arrays, and quantitative PCR in distal CIN colon cancers. Cancer Lett 282: 195-204, 2009.

44. Tanikawa C, Kamatani $\mathrm{Y}$, Toyoshima O, Sakamoto $\mathrm{H}$, Ito H, Takahashi A, Momozawa Y, Hirata M, Fuse N, Takai-Igarashi T, et al: Genome-wide association study identifies gastric cancer susceptibility loci at 12q24.11-12 and 20q11.21. Cancer Sci 109: 4015-4024, 2018

45. Jang SH, Park JW, Kim HR, Seong JK and Kim HK: ADRM1 gene amplification is a candidate driver for metastatic gastric cancers. Clin Exp Metastasis 31: 727-733, 2014

46. Tsai KW, Lo YH, Liu H, Yeh CY, Chen YZ, Hsu CW, Chen WS and Wang JH: Linc00659, a long noncoding RNA, acts as novel oncogene in regulating cancer cell growth in colorectal cancer. Mol Cancer 17: 72, 2018.

This work is licensed under a Creative Commons Attribution-NonCommercial-NoDerivatives 4.0 International (CC BY-NC-ND 4.0) License. 